\title{
Avaliação de perfil longitudinal de pavimento de concreto de cimento Portland com perfilômetro inercial a laser
}

\author{
Rodrigo Fábio Silva de Oliveira ${ }^{1}$ e Fernando Silva Albuquerque ${ }^{2}$
}

\begin{abstract}
Resumo: A avaliação do perfil longitudinal em pavimentos de Concreto de Cimento Portland é de suma importância, tanto para a o controle da qualidade de serviços executados durante períodos de obra, quanto para monitorar a evolução da irregularidade ao longo de sua vida de serviço. O Perfilômetro Inercial a Laser apresenta grande potencial de uso para este fimm, pois proporciona elevada produtividade e resolução menor que $5 \mathrm{~mm}$ (a depender da velocidade de uso). Nesta pesquisa foram tomados como caso de estudo 09 segmentos da rodovia BR-101/SE, com níveis diferentes de qualidade de perfil. Os perfilogramas e resultados de IP (índices de perfis) e IRI (international roughness index) obtidos com o Perfilômetro Inercial a Laser e Perfilógrafo Califórnia (Equipamento de referência) foram comparados para todos os segmentos estudados. Para o pavimento em estudo, concluiu-se que os perfilogramas obtidos representaram de forma graficamente análoga os defeitos que interferem na irregularidade longitudinal. Na comparação para os índices IP e IRI resultantes dos dois equipamentos, os resultados apresentaram-se estatisticamente semelhantes. Portanto, observa-se que o equipamento Perfilômetro Inercial a Laser representa bem as características dos defeitos que influenciam na irregularidade longitudinal dos pavimentos de Concreto de Cimento Portland. Ele pode ser utilizado para medições do Perfil Longitudinal e obtenção dos índices de irregularidade para aceitação de obras e monitoramento da irregularidade longitudinal nas rodovias em operação.
\end{abstract}

Palavras-chave: perfil longitudinal; pavimento de concreto; perfilômetro inercial a laser.

\begin{abstract}
The evaluation of the longitudinal profile in Portland Cement Concrete Pavements is very important, both for the quality control of roadworks, and to monitor the growth of roughness throughout its life cycle. The Inertial Laser Profilometer can be taken into account for this purpose. It provides high productivity and resolution less than $5 \mathrm{~mm}$ (depending on the speed). In this work, 09 segments of the BR-101/SE highway had taken as study case, all of them with different levels of profile quality. The profilograms and results of PI (profile index) and IRI (international roughness index) computed from the Inertial Laser Profilometer and California Profilograph (reference equipment) were compared for all the segments. From the study case, it was concluded that the profilograms have similar trend and they detect the same distresses that cause changes in roughness. The PI and IRI indexes from both equipments were statistically similar. Therefore, the Inertial Laser Profilometer is a great device to detect distresses that cause roughness in Portland Cement Concrete pavements. The device can be taken into account for longitudinal profile measurements and to evaluate roughness indexes for roadwork acceptance and monitoring IRI in the operating road.
\end{abstract}

Keywords: longitudinal profile; concrete pavement; inertial laser profilometer.

\section{INTRODUÇÃO}

Quando uma rodovia é construída, espera-se que a mesma apresente condições de segurança e conforto ao rolamento adequado para os usuários ao longo da sua vida útil. De acordo com Bowman et. al. (2002), durante muitos anos, a qualidade de rolamento da superfície da rodovia não era medida e o pavimento era considerado aceitável se pudesse ser utilizado. Com o aumento do volume de veículos e suas velocidades, construir pavimentos com melhor qualidade tornou-se uma necessidade.

Embora alguns equipamentos para avaliação de perfil de pavimentos já existissem desde a década de 1920 (BERNUCCI et al., 2006), o primeiro método para quantificar, de forma sistemática, o conforto ao rolamento em vias pavimentadas foi o da serventia, desenvolvido na década de 1960 por Carey e Irick (1960) para as pistas experimentais da AASHTO.

\footnotetext{
${ }^{1}$ Rodrigo Fábio Silva de Oliveira, Departamento de Engenharia Civil, UFS. (rodrigofabiooliveira@gmail.com)

2 Fernando Silva Albuquerque, Departamento de Engenharia Civil, UFS. (albuquerque.f.s@uol.com.br)
}

Manuscrito recebido em 20/04/2016 e aprovado para publicação em $08 / 11 / 2016$.

Este artigo é parte de TRANSPORTES v. 25, n. 1, 2017. ISSN: 2237-1346 (online). DOI:10.14295/transportes.v25i1.1138
O conforto ao rolamento está diretamente ligado ao perfil longitudinal da superfície do pavimento, pois os defeitos contidos neste irão transmitir desconforto ao usuário em forma de vibrações no veículo, podendo ser quantificado de forma subjetiva, através de conceitos atribuídos, conforme a norma DNIT 063/2004 - PRO, e de forma objetiva, quantificando índices de qualidade de perfil, conforme a norma DNIT 062/2004 - PRO, obtidos através de medições do perfil longitudinal da rodovia. Diversos índices foram desenvolvidos para esta quantificação, entre eles os mais utilizados são o International Roughness Index (IRI), Índice de Perfil (IP), Ride Number (RN) e Quociente de Irregularidade $(\mathrm{QI})$.

A irregularidade do pavimento pode impactar negativamente várias características funcionais e operacionais da rodovia. Talvez seja considerado o mais importante indicador de desempenho dos pavimentos, pois afeta diretamente o conforto e a segurança aos usuários da rodovia (PATERSON, 1987; CANTISANI E LOPRENCIPE, 2010; SONCIM et. al., 2013). De acordo com Paterson (1987), a irregularidade longitudinal afeta a dinâmica dos veículos e por isso tem um impacto importante em seu custo operacional, na segurança, no conforto e na velocidade do deslocamento. Além disso, também aumenta as cargas dinâmicas aplicadas pelos veículos no pavimento, acelerando sua deterioração 
estrutural e, por fim, pode ainda prejudicar a drenagem superficial, o que tende a aumentar ainda mais seus impactos negativos aos usuários.

Benevides e Motta (2010) destacam a importância da velocidade do veículo no grau de percepção da irregularidade do pavimento pelo usuário, e que as distorções que originam as irregularidades têm grande influência no custo de operação dos veículos.

Medidas de irregularidade longitudinal para a aceitação de construção geralmente são realizadas logo após a pavimentação concluída, utilizando um perfilógrafo ou um perfilômetro inercial. Os resultados obtidos nas medições são usados para avaliar se o pavimento recém-construído alcançou o nível de irregularidade abaixo do máximo especificado (KSAIBATI e ADKINS, 1993).

Segundo Divinsky (2000), as características da irregularidade longitudinal são variáveis, e o método de obtenção das medições, bem como as especificações dos equipamentos, exerce efeito significativo sobre o tratamento e interpretação dos resultados.

Para que a determinação do índice de irregularidade seja representativa, é necessário medir um perfil longitudinal que apresente uma boa relação com o perfil verdadeiro, e que descreva bem as características dos defeitos que exercem influência na determinação do índice de irregularidade escolhido (BARELLA, 2008).

Existem diversos tipos de equipamentos que realizam medições do perfil longitudinal. Sendo distinguidos em função do tipo e método utilizado para o levantamento, estes equipamentos têm sido classificados como de avaliação direta, quando são capazes de medir perfis longitudinais com ou sem contato com o pavimento, e de avaliação indireta, quando fornecem índices de irregularidade a partir da reação de um dispositivo (ou suspensão do veículo) quando trafegando sobre o pavimento (SAYERS et. al., 1986; SAYERS e KARAMIHAS,1998; BOTTURA, 1998).

A norma brasileira vigente para a medição de perfil longitudinal de pavimentos rígidos determina o emprego do Perfilógrafo Califórnia para determinação do Índice de Perfil (IP) (DNIT 049/2013-ES). Contudo, a norma cita que, opcionalmente, o acabamento longitudinal da superfície pode ser verificado por outro dispositivo equivalente para esta finalidade, desde que previamente aprovado pelo Instituto de Pesquisas Rodoviárias (IPR).

A pouca disponibilidade de equipamentos do tipo Perfilógrafo Califórnia no Brasil, torna insuficiente o acompanhamento das obras em execução e monitoramento das rodovias em operação. Portanto, o uso de equipamentos com outras tecnologias, mas que atendam às especificações mínimas para obtenção de perfis longitudinais, talvez seja uma opção para a execução destas atividades.

Neste trabalho, dentre os vários índices existentes para quantificar a irregularidade longitudinal, foram abordados os Índices IP (Índice de Perfil) e IRI (International Roughness Index), obtidos através dos equipamentos Perfilógrafo Califórnia e Perfilômetro Inercial a Laser, em 09 segmentos de uma rodovia em Pavimento de Concreto de Cimento Portland (CCP). O objetivo deste estudo foi avaliar a aplicabilidade do Perfilômetro Inercial a Laser para medição de perfis longitudinais destes tipos de pavimentos.

\section{IRREGULARIDADE DE SUPERFÍCIE - PAVIMENTOS DE CONCRETO}

Vários estudos têm abordado que a irregularidade inicial do pavimento afeta muito a progressão da irregularidade ao longo do tempo (WEN e CHEN, 2007; WEN et. al. 2005; SMITH et. al. 1996). Lee (2007) relata que a baixa qualidade ao rolamento inicial leva o pavimento a elevados custos de reabilitação e menor vida útil.

Wen e Chen (2007) analisaram os possíveis fatores de projeto e construção que afetam a irregularidade longitudinal inicial no pavimento de concreto e concluíram que as placas de concreto mais espessas resultam em irregularidade inicial menor do que as placas de concreto com menor espessura, e que não há diferença estatisticamente significativa resultante dos diferentes métodos de inserção de barras e espaçamento entre as juntas. De acordo com Liu et. al. (2008), juntas defeituosas causam irregularidade no pavimento de concreto, reduzindo sua qualidade ao rolamento. Perera e Kohn (2005), concluíram que pavimentos com barras de transferência fixadas à base apresentavam irregularidades menores do que os pavimentos com as barras inseridas.

Siddique et al. (2007) pesquisaram sobre o efeito da cura no desenvolvimento da irregularidade e relataram que, apesar do elevado gradiente de temperatura $\left(24^{\circ} \mathrm{C}\right)$ entre o topo e a base da placa de CCP recém-construída, a aplicação do composto agente de cura reduz significativamente a irregularidade logo após a conclusão da construção e ao longo do tempo. Kim et. al. (2008) relatam que os índices de irregularidade longitudinal analisados com os dados obtidos no período matutino apresentaram pequenas diferenças aos índices obtidos com os dados do período vespertino.

De acordo com Chou e Pellinen (2005), o fator que mais afetou a irregularidade nos pavimentos de CCP analisados ao longo do tempo foi o seu valor inicial, adquirido após execução. Uma elevada irregularidade inicial para um pavimento de CCP é extremamente indesejável, pois produzirá um mau desempenho ao pavimento em longo prazo (KSAIBATI e ADKINS, 1993).

Para Nakahara e Balbo (2006), não existe um mecanismo principal que possa ser atribuído à alteração da irregularidade do pavimento, ou seja, os diferentes defeitos combinados contribuem para o seu aumento.

\section{ESTUDO DO PERFIL LONGITUDINAL}

De acordo com Sayers e Karamihas (1998), o perfil é uma fatia bidimensional da superfície de uma via, tomada ao longo de uma linha imaginária. Segundo Wang (2006), um perfil de pavimento representa as elevações verticais da superfície do pavimento como uma função da distância longitudinal ao longo de um caminho percorrido.

$O$ perfil de uma rodovia pode ser medido no sentido longitudinal e/ou transversal, que representam a elevação da superfície de rolamento e a inclinação transversal, respectivamente, em relação a um plano de referência e no sentido do tráfego.

Segundo Barella (2008), é correto afirmar que só existe um perfil verdadeiro em cada linha imaginária que se escolha, e por isso, normalmente, duas medições de perfil longitudinal só serão iguais se forem realizadas exatamente 
sobre a mesma linha imaginária, a menos que se esteja diante de uma pista perfeitamente plana. Para que os índices de conforto ao rolamento representem uma boa relação com o perfil verdadeiro do pavimento, é necessário realizar uma medição do perfil longitudinal eficiente e confiável. Quanto mais preciso o equipamento, a medição mais se aproxima do perfil verdadeiro. Então uma boa medição do perfil longitudinal é a que apresenta as características necessárias para a identificação dos defeitos que causam o desconforto ao rolamento.

Segundo Paterson (1987), os desvios da superfície no perfil longitudinal tendem a ser aleatórios, mas podem ser convenientemente caracterizados por uma combinação de formas de onda com diferentes amplitudes e comprimentos.

A classificação de textura proposta pela PIARC (The World Road Association) leva em consideração justamente o comprimento de onda da medição realizada. De acordo com esta classificação, pode-se obter informações relativas à superfície de um pavimento, como microtextura e macrotextura (relacionadas à aderência e drenagem superficial conferida), e a megatextura e irregularidade (relacionadas à resistência ao rolamento, derivadas dos defeitos de superfície e da dinâmica veicular) (SANDBERG E EJSMONT, 2002).

A importância de que o perfil longitudinal seja representativo do perfil real se dá além da quantificação dos índices de conforto, pois os defeitos encontrados como bumps (saliências) e depressões, podem ser localizados em campo de forma precisa para que se proceda aos eventuais trabalhos corretivos. Isto é possível quando, a partir das medições do perfil superficial, obtém-se um gráfico chamado perfilograma, representado no eixo das ordenadas pela elevação na superfície do pavimento (relativa ao perfil ideal) e no eixo das abscissas a distância percorrida. Deste mesmo gráfico pode-se obter o chamado Índice de Perfil (IP), que retrata a qualidade do perfil longitudinal.

\section{1. Índice de Perfil (IP)}

Segundo a CALIFORNIA TEST 526 (2012) o índice de Perfil (IP) é expresso como uma unidade de medida linear que representa o excesso da blanking band. Segundo a ASTM E1274-03 (ASTM, 2012), a blanking band é uma faixa posicionada longitudinalmente ao pavimento, com amplitudes iguais (abaixo e acima) ao longo da linha que representa o perfil longitudinal otimizado.

Não existe um padrão mundial para cálculo do IP a partir do perfilograma. Cada agência dos EUA tem os seus próprios procedimentos padronizados (PERERA e KOHN, 2002; KSAIBATI e ADKINS, 1993).

De acordo com a norma ASTM E2955-13 (ASTM, 2014), para o cálculo do IP, as magnitudes das irregularidades identificadas acima ou abaixo da blanking band no perfilograma são anotadas junto a elas. O cálculo do IP, para cada trecho, é realizado conforme a equação (1):

$$
I_{p}=\frac{\sum h}{L} \times 1000
$$

Onde: $I p=$ Índice de Perfil $(\mathrm{mm} / \mathrm{km})$;

$\sum h=$ Soma das magnitudes das irregularidades no trecho (mm); e
$L=$ Comprimento do trecho $(\mathrm{m})$.

É importante destacar que o cálculo do IP pode ser afetado significativamente pela precisão do levantamento, ou pela inclusão no cálculo de pequenos desvios causados por pedras, textura em excesso, sujeira ou acúmulo de água no percurso do equipamento de medição de perfil. Estas interferências e pequenos obstáculos perdem sua importância quando, a partir do perfil longitudinal, obtêm-se o chamado International Roughness lndex (IRI).

\subsection{International Roughness Index (IRI)}

Este índice surgiu a partir da pesquisa internacional de avaliação de pavimentos, coordenado pelo Banco Mundial com participação dos Estados Unidos, França, Bélgica, Reino Unido e ampla participação do Brasil. O estudo, após analisar vários possíveis índices, escolheu o International Roughness lndex, ou simplesmente IRI, que simula a resposta dinâmica de um veículo de referência, quando trafegando sobre o perfil longitudinal medido (SAYERS e KARAMIHAS, 1998). De acordo com Wang (2006), o IRI é o índice de irregularidade mais utilizado nos EUA.

A norma ASTM E1926 define o procedimento padrão para a o cálculo do IRI a partir de medições do perfil longitudinal. Este cálculo é baseado em um modelo matemático, chamado de modelo de quarto-de-carro (ASTM, 1999a).

Segundo Barella (2008), o IRI representa a passagem de um modelo de quarto-de-carro com parâmetros preconcebidos com um perfil longitudinal, podendo-se dizer que tal modelo funciona como um filtro que retira do "sinal" determinados comprimentos de onda menores que um determinado valor e maiores que outros.

O quarto-de-carro é movido ao longo do perfil longitudinal a uma velocidade de simulação de $80 \mathrm{~km} / \mathrm{h}$. O modelo matemático calcula a deflexão da suspensão do quartode-carro utilizando os deslocamentos do perfil medido e parâmetros da estrutura do carro. A suspensão de movimento simulado é acumulada e, em seguida, dividida pela distância percorrida para se obter um índice com a unidade de inclinação $(\mathrm{m} / \mathrm{km})$, que é chamado de IRI.

\section{EQUIPAMENTOS AVALIADOS NO ESTUDO DE PERFIL LONGITUDINAL}

Benevides e Motta (2010) citam que, devido à importância de mensurar as irregularidades longitudinais dos pavimentos, diversos equipamentos podem ser utilizados para esta finalidade. Os equipamentos medidores de irregularidades diferem uns dos outros quanto às características, à produtividade e à eficiência do levantamento.

Para atender ao objetivo deste trabalho, foram selecionados dois equipamentos de medição direta de perfil longitudinal. O primeiro, objeto do presente estudo, foi o Perfilômetro Inercial a Laser. O segundo foi o Perfilógrafo Califórnia, equipamento padrão para medição de perfis longitudinais em pavimentos de CCP, que serviu para validar as medições realizadas com o primeiro equipamento. 


\subsection{Perfilógrafo Califórnia}

O perfilógrafo Califórnia consiste em uma estrutura montada em forma de uma treliça metálica com comprimento de 7,62 m, suportada sobre um conjunto de rodas em ambas as extremidades.

$\mathrm{O}$ equipamento registra o perfil da superfície do pavimento a partir do movimento vertical de uma roda fixada à armação no ponto médio (perfil da roda), que é referência para a elevação média dos pontos de contato com a superfície da via, estabelecida pelos conjuntos de rodas de suporte (CALIFORNIA TEST 526, 2012).

Os perfilógrafos são conhecidos por amplificar e atenuar comprimentos de ondas em intervalos distintos, influenciando no perfil da superfície do pavimento medido. Devido a isto têm sido levantadas preocupações sobre a adequação do uso de dados obtidos com perfilógrafos para avaliar a irregularidade longitudinal para a aceitação de pavimentos recém-construídos (KSAIBATI e ADKINS, 1993; SMITH et al., 2002).

De qualquer forma, o perfilógrafo é ainda o equipamento mais comumente utilizado para a medição de irregularidade em pavimentos de concreto de cimento Portland nos EUA, sendo normatizado, por exemplo, pela ASTM E1274-03 (2012) e pela CALIFORNIA TEST 526 (2012).

Segundo Barella (2008), o perfilógrafo é tão popular porque fornece uma visão rápida da irregularidade longitudinal do pavimento, e os resultados apontam visivelmente, de forma gráfica, os locais onde há necessidade de trabalho corretivo, com pequeno erro. Entretanto, este equipamento apresenta baixa produtividade devido a sua força motriz ser manual.

Observa-se na Figura 1 o perfilógrafo Califórnia utilizado para os levantamentos deste trabalho.

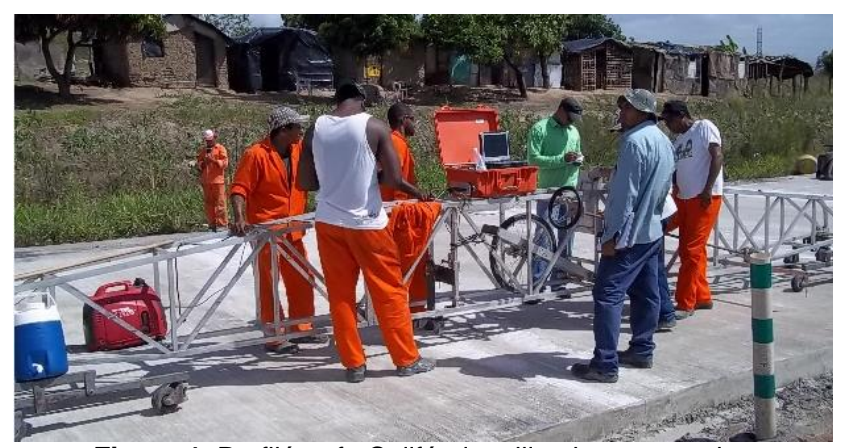

Figura 1. Perfilógrafo Califórnia utilizado na pesquisa

\subsection{Perfilômetro Inercial a Laser}

Trata-se de um equipamento destinado à medida do perfil longitudinal de um pavimento que possui um componente inercial (acelerômetro medidor da aceleração vertical), destinado à compensação dos movimentos verticais do veículo (BARELLA, 2008).

O Perfilômetro Inercial a Laser utilizado nesta pesquisa é de propriedade da Universidade Federal de Sergipe (UFS) e foi desenvolvido no Brasil (BARELLA, 2008). O equipamento é modular (Figura 2), composto por 3 módulos a laser, 2 acelerômetros gravitacionais, um hodômetro e um GPS, permitindo o acréscimo ou a supressão de módulos lasers, conforme a necessidade de cada levantamento. Este equipamento possui elevada taxa de aquisição de dados (4000 pontos/segundo), o que torna possível a construção de perfis longitudinais com uma nuvem de pontos com espaçamento milimétrico, a depender da velocidade de tráfego. Outras vantagens de uso deste equipamento são: a possibilidade de trafegar na velocidade normal da rodovia; ser utilizado em períodos noturnos ou diurnos etc. O procedimento de medição atendeu às especificações da norma ASTM E950-98 (ASTM, 1999b).

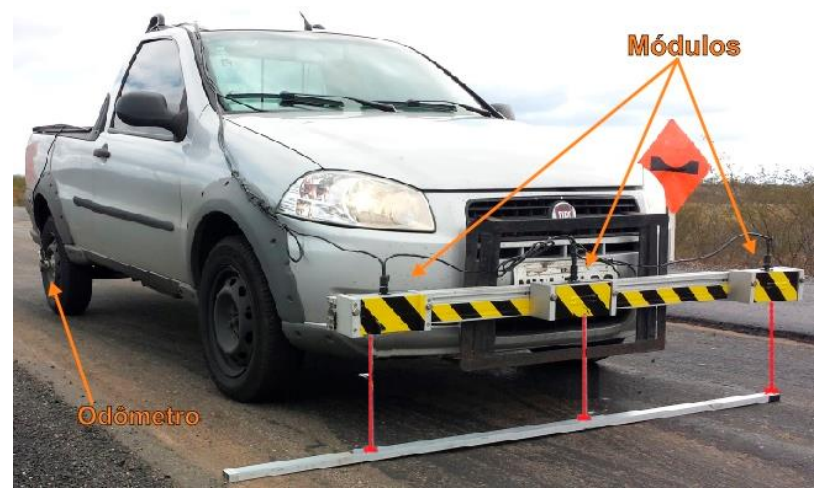

Figura 2. Perfilômetro Inercial a Laser da UFS devidamente acoplado no veículo

\section{MÉTODOS}

A estrutura dimensionada para o pavimento de CCP do trecho experimental apresenta: Sub-base em Concreto Compactado com Rolo (CCR) com $10 \mathrm{~cm}$ de espessura, com resistência característica do concreto à compressão (fck) mínima de $5 \mathrm{MPa}$ aos 7 dias de cura; Placa de Concreto de Cimento Portland (CCP), com $21 \mathrm{~cm}$ de espessura e comprimento máximo de $5,00 \mathrm{~m}$, compostas por barras de transferência e ligação, e concreto com resistência à tração na flexão (fctmk) mínima de 4,5 MPa aos 28 dias de cura. Cabe esclarecer que a camada de CCR certamente exerce a função de base na estrutura deste pavimento, contudo foi definida neste trabalho como sub-base para se adequar à nomenclatura do projeto e também as adotadas pelo Departamento de Infra Estrutura de Transportes (DNIT) e pela Associação Brasileira de Cimento Portland (ABCP).

Para realizar a comparação entre os perfilogramas gerados pelos dois equipamentos estudados, foram procedidas análises visuais dos gráficos e comparações entre o tipo, a localização e magnitude dos defeitos identificados por cada um.

Aplicaram-se também testes de hipóteses aos valores de IP e IRI obtidos a partir dos perfis longitudinais levantados pelos dois equipamentos, verificando a normalidade das distribuições (teste de normalidade Anderson-Darling), a igualdade das variâncias (teste F) e igualdade das médias (teste t) de cada amostra. Isto tornou possível concluir pela similaridade ou não dos resultados dos índices obtidos pelos equipamentos para o pavimento em estudo.

\subsection{Escolha dos Segmentos Analisados}

O perfil longitudinal foi obtido em um trecho contínuo de 2,0 km de comprimento, localizado na BR-101/SE (Figura 3), para as duas faixas de tráfego da rodovia e sobre as duas trilhas de roda, utilizando um Perfilógrafo Califórnia. Após a obtenção dos perfis, foram gerados relatórios contendo a identificação de defeitos (bumps e depressões) e os valores dos Índices de Perfil (IP) para segmentos de comprimento de $100 \mathrm{~m}$. 


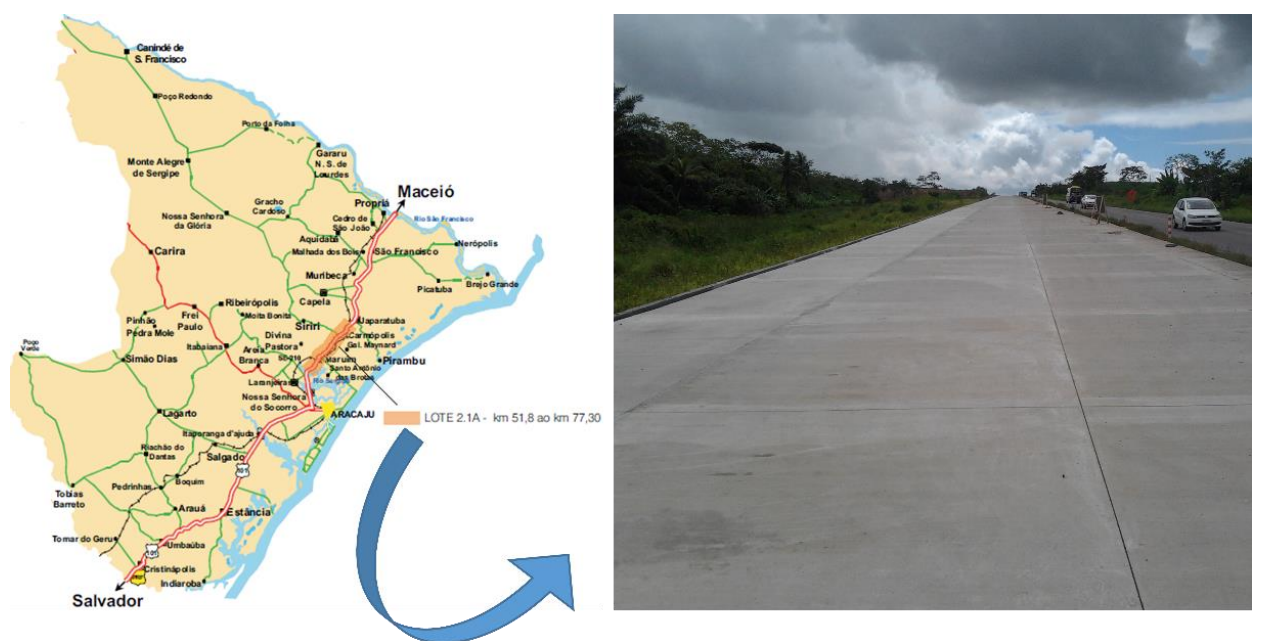

Figura 3. Mapa de localização e vista panorâmica de um trecho estudado

Tabela 1. Segmentos escolhidos para a coleta de dados de perfil longitudinal.

\begin{tabular}{ccccc}
\hline Nível & $\begin{array}{c}\text { Segmento/Trilha de } \\
\text { Roda }\end{array}$ & $\begin{array}{c}\text { Marco inicial } \\
(\mathbf{k m})\end{array}$ & $\begin{array}{c}\text { Marco final } \\
(\mathbf{k m})\end{array}$ & $\begin{array}{c}\text { IP (mm/km) } \\
\text { Perfilógrafo Califórnia }\end{array}$ \\
& $12 / 2$ & 155,52 & 155,62 & 0 \\
Baixo & $12 / 1$ & 155,52 & 155,62 & 13 \\
$(0 \leq \mathrm{IP} \leq 200 \mathrm{~mm} / \mathrm{km})$ & $13 / 1$ & 155,42 & 155,52 & 25 \\
\hline Médio & $5 / 1$ & 156,22 & 156,32 & 368 \\
\multirow{2}{*}{$(200<\mathrm{IP} 400 \mathrm{~mm} / \mathrm{km})$} & $5 / 2$ & 154,82 & 154,92 & 216 \\
& $19 / 2$ & 156,22 & 156,32 & 343 \\
Alto & $23 / 1$ & 154,42 & 154,52 & 414 \\
$(\mathrm{IP}>400 \mathrm{~mm} / \mathrm{km})$ & $17 / 2$ & 156,02 & 156,12 & 724 \\
& $7 / 1$ & 156,02 & 156,12 & 813 \\
\hline
\end{tabular}

Foram selecionados 9 segmentos (amostras) na faixa de rolamento 2 (detalhados na Tabela 1) a partir dos valores de IP obtidos nestes segmentos, representando os intervalos dos seus tercis, com valores abaixo, dentro e acima do terço médio.

\subsection{Tratamento dos dados}

Os perfilogramas e os índices IP e IRI foram gerados com os dados coletados por cada equipamento através do software ProVal 3.5 (Profile Viewing and Analysis). Este software é de domínio público, desenvolvido pelo US Department of Transportation da Federal Highway Administration (FHWA) e Long Term Pavement Performance Program (LTPP), permitindo a visualização e análise dos perfis de várias maneiras diferentes.

De acordo com ASTM E1274-03 (ASTM, 2012), existem diferenças nas respostas de frequência entre perfilógrafos com rodas uniformemente espaçadas e perfilógrafos com rodas não uniformemente espaçadas. Devido a isto, o espaçamento entre as rodas utilizados no software foram idênticos ao espaçamento das rodas do perfilógrafo utilizado para a medição do perfil.

\section{RESULTADOS E DISCUSSÕES}

\subsection{Análise dos Perfilogramas}

Objetivando avaliar comparativamente os perfilogramas obtidos pelos dois equipamentos, foram realizadas análises visuais destes gráficos para cada intervalo de valores de IP, conforme os segmentos selecionados na Tabela 1.

Para representar os segmentos com baixo valor de IP, foi selecionado o segmento $12 / 1(\mathrm{IP}=13 \mathrm{~mm} / \mathrm{km})$ (Figura 4).
Observa-se que os perfilogramas obtidos apresentam representação gráfica similar para os defeitos que exercem influência na irregularidade longitudinal. Observou-se que o pico (um bump) identificado no perfil de referência é reproduzido com formato e localização semelhantes no perfilograma do perfilômetro inercial a laser.

Analisando as magnitudes do pico coincidente nos perfilogramas, nota-se que os valores de $2,50 \mathrm{~mm}$ no Perfilômetro Inercial a Laser e 1,30 mm no Perfilógrafo Califórnia geram um alto desvio percentual $(92,31 \%)$.

Para representar os segmentos com valores médios de IP, foi selecionado o perfilograma obtido para o segmento 19/2 (Figura 5). Também se observa que os gráficos identificam similarmente os defeitos que exercem influência na irregularidade longitudinal. No perfilograma obtido com o perfilógrafo Califórnia foram identificados 4 bumps e 6 depressões, enquanto que no perfilograma obtido com o perfilômetro inercial a laser foram identificados 6 bumps e 6 depressões. Observou-se que os picos identificados pelos equipamentos também são reproduzidos com formato similar, assim como a faixa do perfil que não extrapola o blanking band, com exceção de 2 bumps a mais identificados pelo perfilômetro inercial a laser e 1 depressão a mais identificada por cada um dos equipamentos, mas não coincidentes na comparação entre perfilogramas. Apesar disso, o perfilograma do perfilógrafo Califórnia representa graficamente, dentro dos limites da blanking band, os defeitos identificados no perfilograma do perfilômetro inercial a laser.

Isso leva a concluir que a diferença de representação fora da faixa do blanking band se refere a alterações nos parâmetros de representação do comprimento de onda do defeito. 


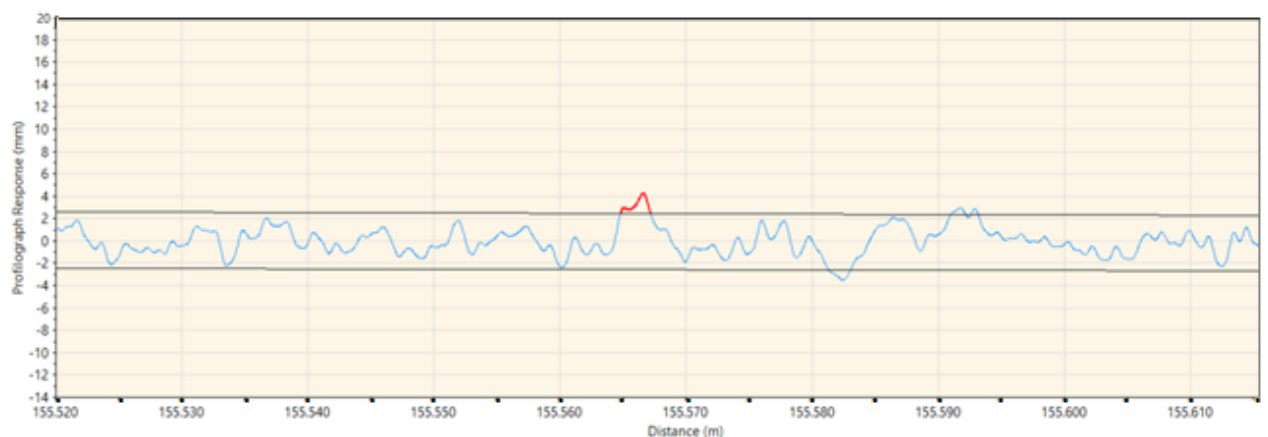

(a)

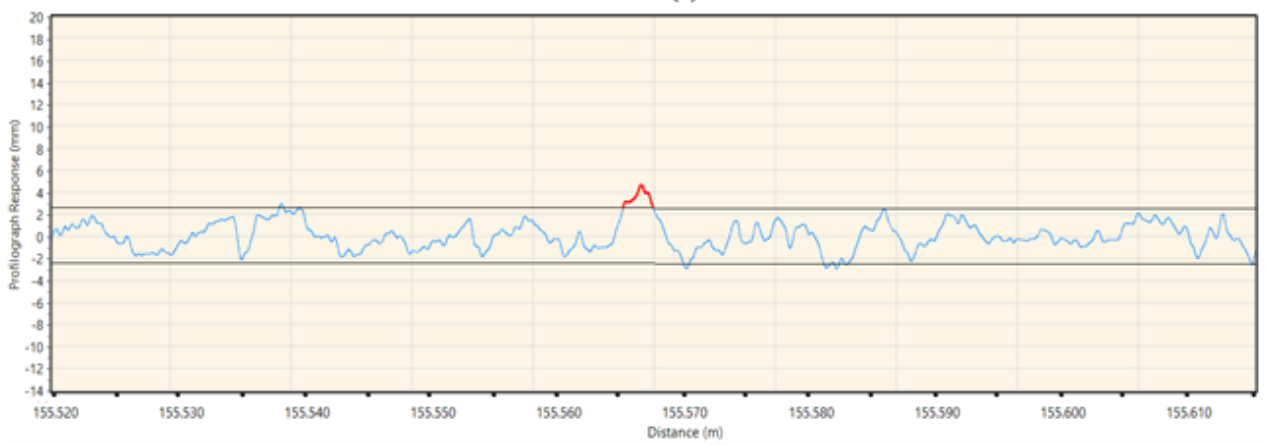

(b)

Figura 4. Comparação de perfilogramas no segmento 12/1: (a) Perfilógrafo Califórnia; (b) Perfilômetro Inercial a Laser.

Provavelmente o perfilômetro inercial a laser encontrou 3 defeitos a mais devido ao maior detalhamento na leitura do perfil longitudinal feita por este equipamento.

A Tabela 2 apresenta o resumo dos defeitos identificados, contendo o tipo do defeito, a localização inicial e final, o valor do pico e os desvios percentuais dos defeitos encontrados no segmento.

Identificam-se seis $(66,67 \%)$ defeitos com desvio percentual elevado para os seus picos e três $(33,33 \%)$ defeitos em comum que não apresentavam desvio percentual (ver Tabela 2). Contudo, é observada uma tendência similar de identificação de picos, porém com maiores magnitudes no perfilograma resultante do perfilômetro inercial a laser.

O segmento 23/1 representa os segmentos com valores altos de IP (Figura 6). Conforme aconteceu nos outros segmentos analisados, há similaridade nos gráficos de defeitos, onde os perfilogramas resultantes de levantamentos com os dois equipamentos são análogos. Observou-se que os picos identificados no perfil de referência também são reproduzidos com formato similar no perfilograma do perfilômetro inercial a laser. Contudo, este último equipamento identificou 3 depressões a mais e 1 bump a menos que o perfilógrafo Califórnia.

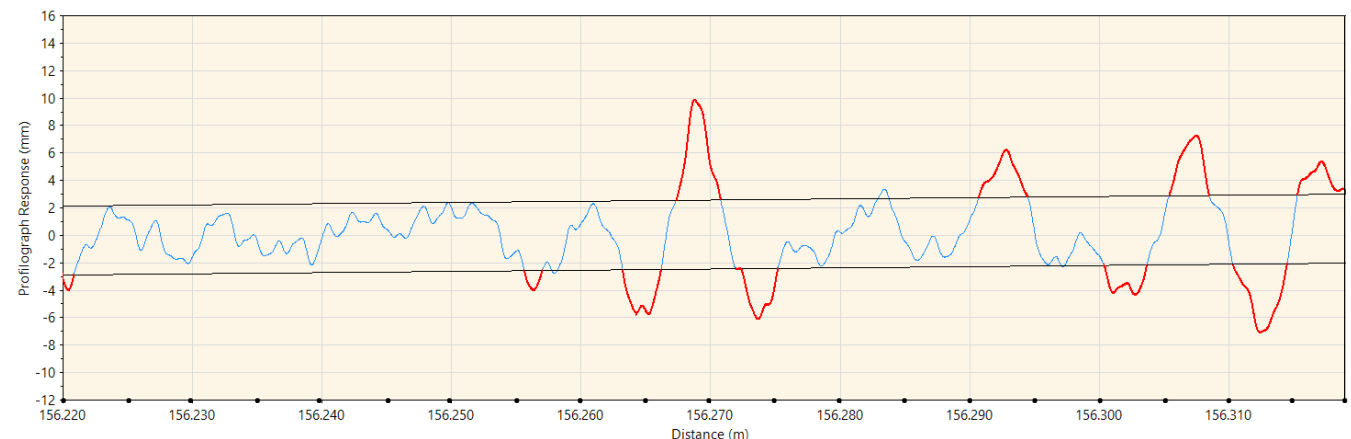

(a)

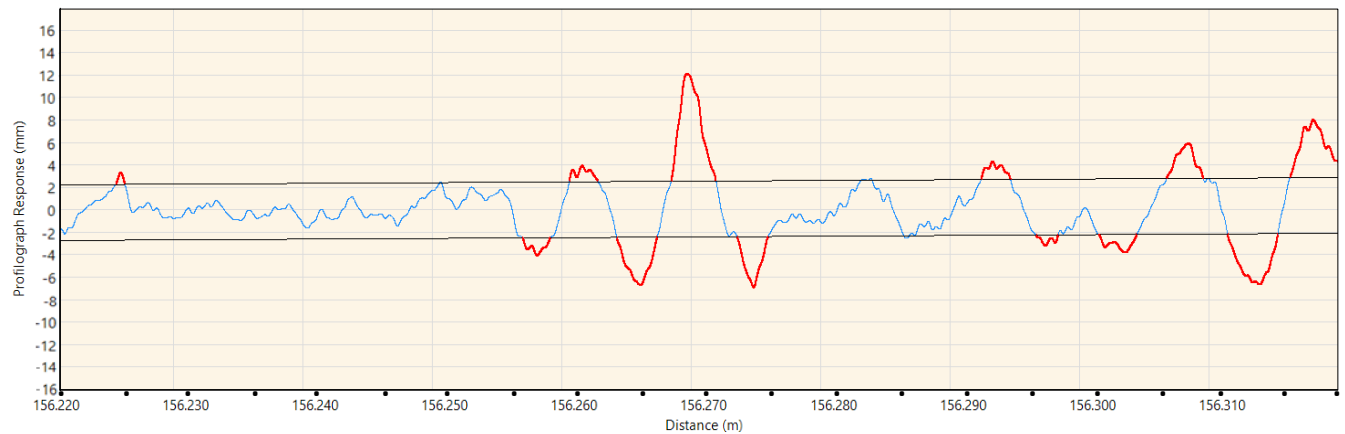

(b)

Figura 5. Comparação de perfilogramas no segmento 19/2: (a) Perfilógrafo Califórnia; (b) Perfilômetro Inercial a Laser 
OLIVEIRA, R.F.S.; ALBUQUERQUE, F.S.

Tabela 2. Resumo da identificação dos defeitos e desvio percentual no segmento 19 FR2 Trilha de roda 2

\begin{tabular}{|c|c|c|c|c|c|c|c|c|c|}
\hline \multirow[b]{2}{*}{$\begin{array}{c}\text { Defeitos } \\
\text { coincidentes }\end{array}$} & \multicolumn{4}{|c|}{ Perfilógrafo Califórnia (PC) } & \multicolumn{4}{|c|}{ Perfilômetro Inercial a Laser (PIL) } & \multirow[b]{2}{*}{$\begin{array}{l}\text { Desvio Percentual } \\
\text { PC x PIL (\%) }\end{array}$} \\
\hline & $\begin{array}{l}\text { Tipo do De- } \\
\text { feito }\end{array}$ & $\begin{array}{c}\text { Distância } \\
\text { Inicial (m) }\end{array}$ & $\begin{array}{l}\text { Distância } \\
\text { Final (m) }\end{array}$ & $\begin{array}{c}\text { Pico } \\
(\mathbf{m m})\end{array}$ & $\begin{array}{l}\text { Tipo do } \\
\text { Defeito }\end{array}$ & $\begin{array}{c}\text { Distância } \\
\text { Inicial (m) }\end{array}$ & $\begin{array}{l}\text { Distância } \\
\text { Final (m) }\end{array}$ & $\begin{array}{l}\text { Pico } \\
(\mathbf{m m})\end{array}$ & \\
\hline & Depressão & 0,00 & 0,80 & 1,30 & & & & & \\
\hline & & & & & Bump & 3,80 & 4,50 & 1,30 & \\
\hline \multirow[t]{2}{*}{1} & Depressão & 35,60 & 37,00 & 1,30 & Depressão & 35,21 & 37,39 & 1,30 & 0,00 \\
\hline & & & & & Bump & 38,83 & 41,05 & 1,30 & \\
\hline 2 & Depressão & 43,20 & 46,20 & 3,80 & Depressão & 42,49 & 45,62 & 3,80 & 0,00 \\
\hline 3 & Bump & 47,30 & 50,80 & 7,60 & Bump & 46,71 & 50,14 & 8,90 & 17,11 \\
\hline 4 & Depressão & 51,90 & 55,20 & 3,80 & Depressão & 51,79 & 54,19 & 5,10 & 34,21 \\
\hline \multirow[t]{2}{*}{5} & Bump & 70,70 & 74,50 & 3,80 & Bump & 70,65 & 72,91 & 1,30 & 65,79 \\
\hline & & & & & Depressão & 74,88 & 76,60 & 1,30 & \\
\hline 6 & Depressão & 80,40 & 83,70 & 2,50 & Depressão & 79,73 & 82,69 & 1,30 & 48,00 \\
\hline 7 & Bump & 85,40 & 88,50 & 3,80 & Bump & 85,00 & 87,79 & 2,50 & 34,21 \\
\hline 8 & Depressão & 90,30 & 94,50 & 5,10 & Depressão & 89,68 & 93,57 & 5,10 & 0,00 \\
\hline 9 & Bump & 95,30 & 99,10 & 2,50 & Bump & 94,51 & 98,25 & 5,10 & 104,00 \\
\hline
\end{tabular}

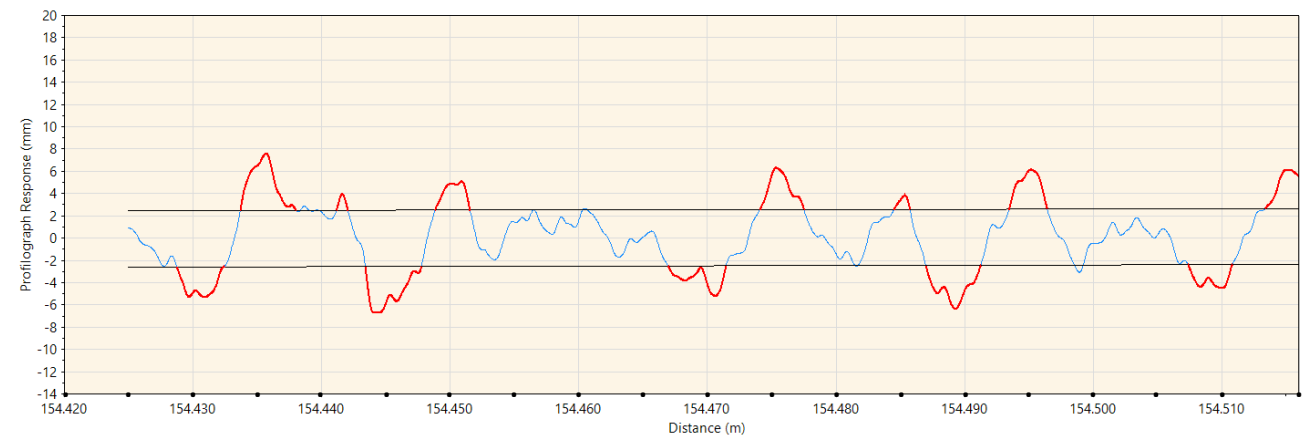

(a)

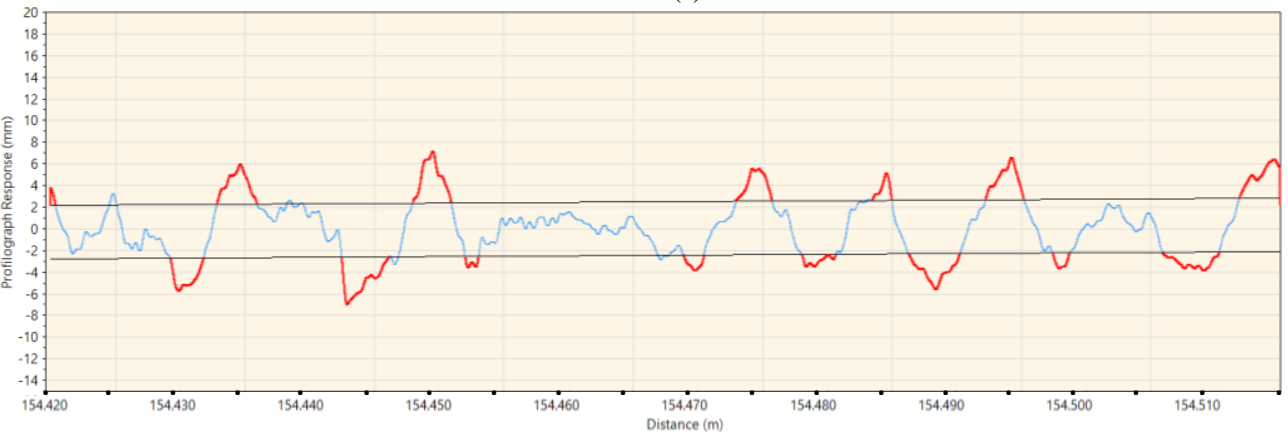

(b)

Figura 6: Comparação de perfilogramas no segmento 23/1: (a) Perfilógrafo Califórnia; (b) Perfilômetro Inercial a Laser

O perfilograma obtido com Perfilógrafo Califórnia identificou 12 defeitos (7 bumps e 5 depressões), enquanto que o perfilômetro inercial a laser identificou 14 defeitos $(6$ bumps e 8 depressões). Pôde-se observar que dentre os defeitos reproduzidos (ver Tabela 3), 11 defeitos são identificados de forma coincidente pelos equipamentos. Destes, 6 $(54,54 \%)$ defeitos apresentaram desvios percentuais elevados para os seus picos e $5(33,33 \%)$ defeitos com desvio percentual nulo.

\subsection{Análise do Índice de Perfil (IP)}

Os índices de Perfil (IP) obtidos para os segmentos estudados estão apresentados na Tabela 4. Observa-se que, em média, o equipamento perfilômetro inercial a laser forneceu valores de IP maiores do que o perfilógrafo Califórnia. Observa-se ainda que os erros percentuais aumentam quanto menores são os valores de IP, com média dos erros absolutos de IP considerados importantes $(57 \mathrm{~mm} / \mathrm{km})$. Esta média foi bastante influenciada pelas diferenças observadas nos IPs dos segmentos 12/1 e 5/2.
A medição do segmento $12 / 2$ teve erro percentual tendendo a infinito, devido o valor de IP resultante do levantamento com o perfilógrafo Califórnia ter sido zero. Para valores de IP de médio a alto, os erros percentuais são menores, indicando que os comprimentos de onda que geram os defeitos com maiores magnitudes são identificados de forma similar pelos equipamentos.

Nos testes de normalidade efetuados, observou-se que as amostragens foram significativas (significâncias superiores ao $\alpha$ de 0,05 ) (Figura 7). Portanto, não se pode rejeitar a hipótese de que as amostras foram retiradas de uma população com distribuição normal (Gauss).

Com esta confirmação, procederam-se os testes $\mathrm{F}$ e $\mathrm{t}$ (Tabela 5). Os $P$-values na comparação proposta na Tabela 5 foram superiores que a significância $\alpha$ de 0,05 . Portanto, não se pode rejeitar a hipótese de igualdade das variâncias (teste F) e da igualdade das médias (teste t) dos valores de IP resultantes dos perfis longitudinais gerados pelos dois equipamentos. 
Tabela 3. Resumo da identificação dos defeitos e desvio percentual no segmento 23 FR2 Trilha de roda

\begin{tabular}{|c|c|c|c|c|c|c|c|c|c|}
\hline \multirow[b]{2}{*}{$\begin{array}{c}\text { Defeitos } \\
\text { coincidentes }\end{array}$} & \multicolumn{4}{|c|}{ Perfilógrafo Califórnia (PC) } & \multicolumn{4}{|c|}{ Perfilômetro Inercial a Laser (PIL) } & \multirow{2}{*}{$\begin{array}{c}\text { Desvio } \\
\text { Percentual } \\
\text { PC x PIL (\%) }\end{array}$} \\
\hline & $\begin{array}{l}\text { Tipo do } \\
\text { Defeito }\end{array}$ & $\begin{array}{c}\text { Distância } \\
\text { Inicial (m) }\end{array}$ & $\begin{array}{l}\text { Distância } \\
\text { Final (m) }\end{array}$ & $\begin{array}{c}\text { Pico } \\
(\mathbf{m m})\end{array}$ & $\begin{array}{l}\text { Tipo do } \\
\text { Defeito }\end{array}$ & $\begin{array}{c}\text { Distância } \\
\text { Inicial (m) }\end{array}$ & $\begin{array}{l}\text { Distância } \\
\text { Final (m) }\end{array}$ & $\begin{array}{c}\text { Pico } \\
(\mathbf{m m})\end{array}$ & \\
\hline 1 & Depressão & 8,70 & 12,40 & 2,50 & Depressão & 9,27 & 11,83 & 2,50 & 0,00 \\
\hline \multirow[t]{2}{*}{2} & Bump & 13,70 & 18,00 & 5,10 & Bump & 12,88 & 15,97 & 3,80 & 25,49 \\
\hline & Bump & 21,10 & 22,00 & 1,30 & & & & & \\
\hline 3 & Depressão & 23,40 & 27,80 & 3,80 & Depressão & 22,51 & 26,15 & 3,80 & 0,00 \\
\hline \multirow[t]{2}{*}{4} & Bump & 28,80 & 31,50 & 2,50 & Bump & 28,00 & 30,98 & 5,10 & 104,00 \\
\hline & & & & & Depressão & 32,06 & 33,09 & 1,30 & \\
\hline 5 & Depressão & 46,90 & 51,50 & 2,50 & Depressão & 48,92 & 50,51 & 1,30 & 48,00 \\
\hline \multirow[t]{2}{*}{6} & Bump & 54,10 & 57,50 & 3,80 & Bump & 53,02 & 55,77 & 2,50 & 34,21 \\
\hline & & & & & Depressão & 58,07 & 60,70 & 1,30 & \\
\hline 7 & Bump & 64,50 & 65,80 & 1,30 & Bump & 63,50 & 65,03 & 2,50 & 92,31 \\
\hline 8 & Depressão & 67,00 & 71,30 & 3,80 & Depressão & 66,32 & 70,29 & 3,80 & 0,00 \\
\hline \multirow[t]{2}{*}{9} & Bump & 73,50 & 76,40 & 3,80 & Bump & 72,29 & 75,23 & 3,80 & 0,00 \\
\hline & & & & & Depressão & 77,45 & 78,78 & 1,30 & \\
\hline 10 & Depressão & 87,40 & 90,80 & 2,50 & Depressão & 85,96 & 90,33 & 1,30 & 48,00 \\
\hline 11 & Bump & 93,40 & 96,90 & 3,80 & Bump & 91,90 & 95,06 & 3,80 & 0,00 \\
\hline
\end{tabular}

Tabela 4. Resumo dos dados de IP e IRI obtidos nos segmentos pelos equipamentos estudados

\begin{tabular}{|c|c|c|c|c|c|c|c|c|c|}
\hline \multirow[b]{2}{*}{ Nível } & \multirow[b]{2}{*}{ Segmento/Trilha } & \multirow[b]{2}{*}{$\begin{array}{c}\text { Marco inicial } \\
(\mathbf{k m})\end{array}$} & \multirow[b]{2}{*}{$\begin{array}{c}\text { Marco final } \\
\quad(\mathbf{k m})\end{array}$} & \multicolumn{3}{|c|}{ IP $(\mathbf{m m} / \mathbf{k m})$} & \multicolumn{3}{|c|}{ IRI $(\mathbf{m} / \mathbf{k m})$} \\
\hline & & & & PIL & $\begin{array}{c}\text { Erro } \\
(\%) \\
\leftarrow \rightarrow\end{array}$ & PC & PIL & $\begin{array}{c}\text { Erro } \\
(\%) \\
\leftarrow \rightarrow\end{array}$ & PC \\
\hline \multirow{3}{*}{ Baixo } & $12 / 2$ & 155,52 & 155,62 & 25 & $\infty$ & 0 & 1,69 & 9,63 & 1,87 \\
\hline & $12 / 1$ & 155,52 & 155,62 & 25 & $\begin{array}{c}100,0 \\
0\end{array}$ & 13 & 1,45 & 13,17 & 1,67 \\
\hline & $13 / 1$ & 155,42 & 155,52 & 38 & 50,00 & 25 & 1,66 & 8,79 & 1,82 \\
\hline \multirow{3}{*}{ Médio } & $5 / 1$ & 156,22 & 156,32 & 290 & 21,23 & 368 & 1,63 & 11,41 & 1,84 \\
\hline & $5 / 2$ & 154,82 & 154,92 & 457 & $\begin{array}{c}111,7 \\
6\end{array}$ & 216 & 2,54 & 19,25 & 2,13 \\
\hline & $19 / 2$ & 156,22 & 156,32 & 328 & 4,47 & 343 & 1,74 & 16,35 & 2,08 \\
\hline \multirow{3}{*}{ Alto } & $23 / 1$ & 154,42 & 154,52 & 414 & 0,00 & 414 & 2,08 & 8,77 & 2,28 \\
\hline & $17 / 2$ & 156,02 & 156,12 & 800 & 10,53 & 724 & 2,78 & 8,17 & 2,57 \\
\hline & $7 / 1$ & 156,02 & 156,12 & 762 & 6,25 & 813 & 2,54 & 4,87 & 2,67 \\
\hline
\end{tabular}

\subsection{Análise do International Roughness Index (IRI)}

Os valores de IRI obtidos com os equipamentos para os segmentos estudados estão apresentados na Tabela 4. O perfil longitudinal do equipamento perfilógrafo Califórnia apresenta em média valores de IRI superiores aos do perfilômetro inercial a laser. Os erros percentuais entre valores de IRI resultantes dos perfis longitudinais obtidos com os dois equipamentos foram maiores para a faixa de valores

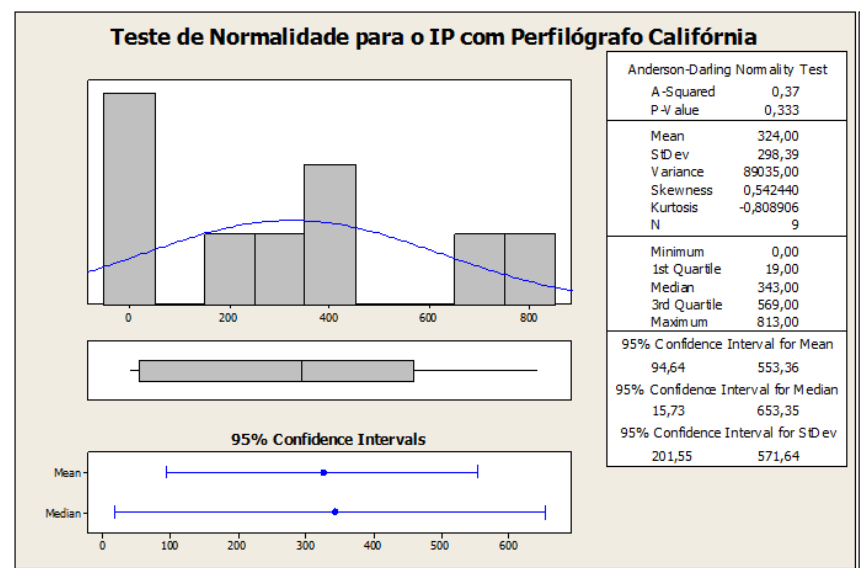

médios. Vale observar que, em geral, a média dos erros absolutos foi elevada $(0,23 \mathrm{~m} / \mathrm{km})$, devido, principalmente, as diferenças de IRI observadas nos segmentos de valores médios.

Observa-se na Tabela 4 que os valores de IRI resultantes do perfilômetro inercial a laser tiveram mesma tendência de crescimento quando comparados com os obtidos a partir do perfilógrafo Califórnia.

Nos testes de normalidade efetuados (Figura 8), observou-se que nas duas amostragens os $P$-values unicaudais foram superiores à significância $\alpha$ de 0,05 .

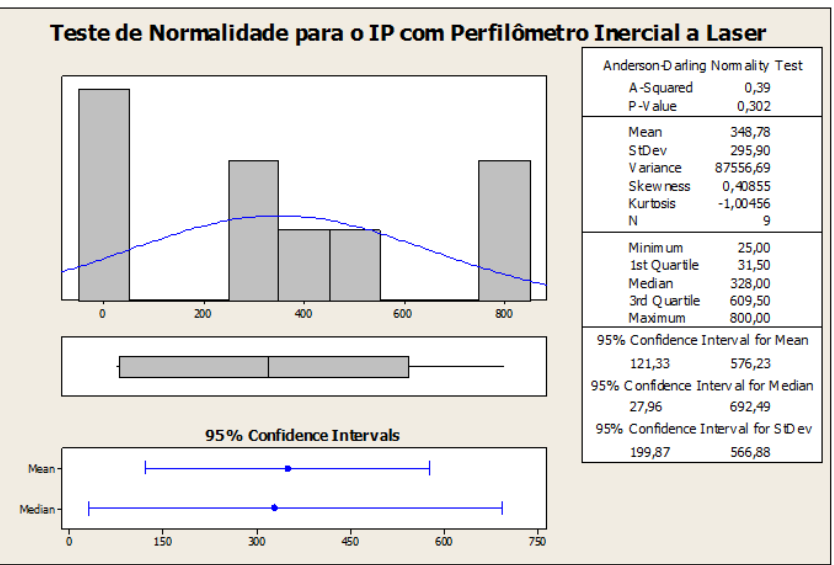

Figura 7. Teste de Normalidade para o IP com o Perfilógrafo Califórnia e com o Perfilômetro Inercial a Laser. 
OLIVEIRA, R.F.S.; ALBUQUERQUE, F.S.

Tabela 5. Resultados do teste-F e do teste-t para as amostragens de IP

\begin{tabular}{|c|c|c|c|c|c|c|c|c|c|}
\hline \multirow{2}{*}{$\begin{array}{c}\text { Média } \\
(\mathbf{m m} / \mathbf{k m})\end{array}$} & \multirow{2}{*}{ Variância } & \multicolumn{4}{|c|}{$\begin{array}{c}\text { Teste } \mathbf{F} \text { - duas amostras } \\
\text { variâncias iguais }\end{array}$} & \multicolumn{4}{|c|}{$\begin{array}{l}\text { Teste } t \text { - duas amostras com } \\
\text { médias de população iguais }\end{array}$} \\
\hline & & $\begin{array}{l}\mathbf{N}^{0} \text { de } \\
\text { obs. }\end{array}$ & $\begin{array}{c}\text { Graus de } \\
\text { lib. }\end{array}$ & $\mathbf{F}$ & $\boldsymbol{P}_{\text {value }}$ & $\begin{array}{c}\mathbf{N}^{\circ} \text { de } \\
\text { obs. }\end{array}$ & $\begin{array}{l}\text { Graus de } \\
\text { lib. }\end{array}$ & Stat t & $\boldsymbol{P}_{\text {value }}$ \\
\hline 324 & 89005 & 9 & 8 & \multirow[b]{2}{*}{1,017} & \multirow[b]{2}{*}{0,491} & 9 & \multirow[b]{2}{*}{16} & \multirow[b]{2}{*}{$-0,178$} & \multirow[b]{2}{*}{0,431} \\
\hline 349 & 87505 & 9 & 8 & & & 9 & & & \\
\hline
\end{tabular}

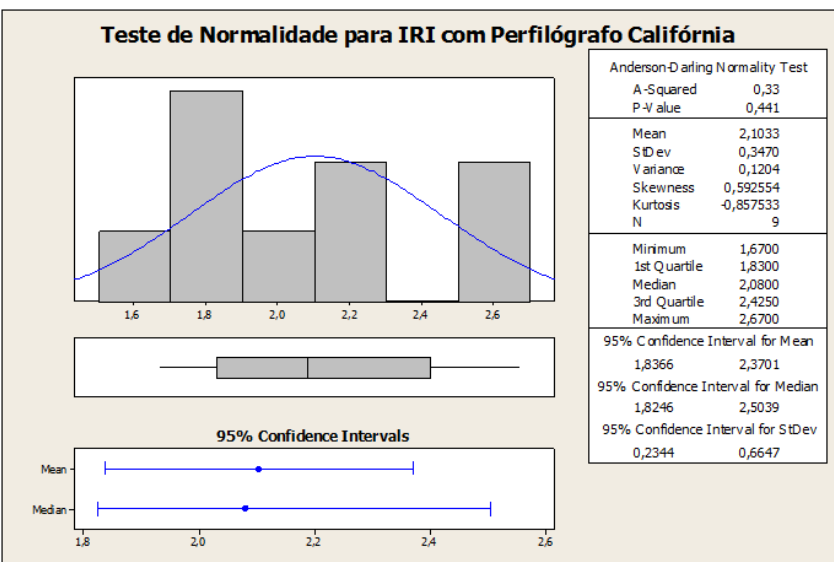

Teste de Normalidade para IRI com Perfilômetro Inercial a Lase

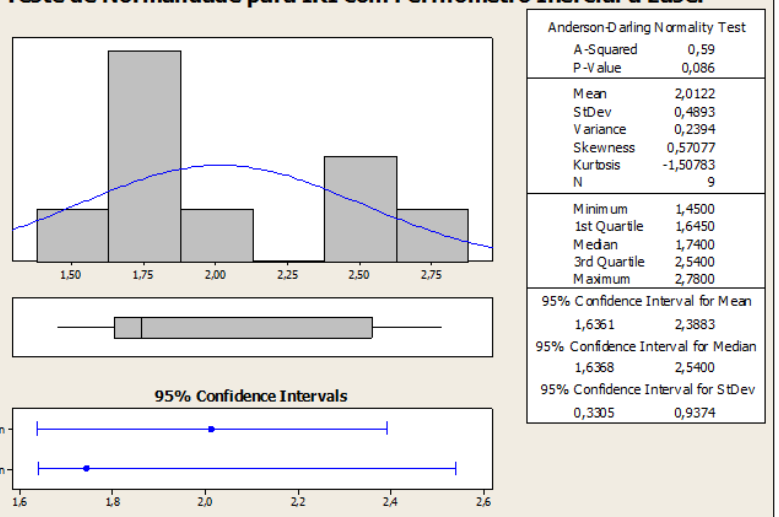

Figura 8. Teste de Normalidade para o IRI com o Perfilógrafo Califórnia e com o Perfilômetro Inercial a Laser

Tabela 6. Resultados do teste-F e do teste-t para as amostragens de IRI

\begin{tabular}{|c|c|c|c|c|c|c|c|c|c|}
\hline \multirow{2}{*}{$\begin{array}{l}\text { Média } \\
(\mathbf{m} / \mathbf{k m})\end{array}$} & \multirow{2}{*}{ Variância } & \multicolumn{4}{|c|}{$\begin{array}{c}\text { Teste } \mathbf{F} \text { - duas amostras } \\
\text { variâncias iguais }\end{array}$} & \multicolumn{4}{|c|}{$\begin{array}{l}\text { Teste } \mathrm{t} \text { - duas amostras com } \\
\text { médias de população iguais }\end{array}$} \\
\hline & & $\begin{array}{l}\mathrm{N}^{0} \text { de } \\
\text { obs. }\end{array}$ & $\begin{array}{l}\text { Graus } \\
\text { de lib. }\end{array}$ & $\mathbf{F}$ & $\boldsymbol{P}_{\text {value }}$ & $\begin{array}{l}\mathrm{N}^{\circ} \text { de } \\
\text { obs. }\end{array}$ & $\begin{array}{l}\text { Graus } \\
\text { de lib. }\end{array}$ & Stat $t$ & $P_{\text {value }}$ \\
\hline 2,10 & 0,12 & 9 & 8 & & & 9 & & & \\
\hline 2,01 & 0,24 & 9 & 8 & 0,503 & 0,175 & 9 & 16 & 0,456 & 0,327 \\
\hline
\end{tabular}

Portanto, não se pode rejeitar a hipótese de que suas amostras foram retiradas de uma população com distribuição normal (Gauss). Procedeu-se os testes F e t (Tabela 6) para verificar se as variâncias e as médias dos valores de IRI obtidos a partir dos dois equipamentos seriam iguais. Os valores de $P$-value unicaudais, obtidos nas comparações apresentadas na Tabela 6 foram superiores que a significância $\alpha$ de 0,05 para o teste-F e o teste-t, não podendo rejeitarse a hipótese de igualdade das variâncias e das médias de IRI.

\section{CONSIDERAÇÕES FINAIS}

Neste trabalho foi avaliada a aplicabilidade do equipamento perfilômetro inercial a laser na medição de perfis longitudinais de pavimentos de concreto de cimento Portland, e consequente obtenção de perfilogramas, Índice de Perfil (IP) e International Roughness Index (IRI). Todos estes resultados foram comparados aos obtidos através do perfilógrafo Califórnia.

Para os perfis longitudinais obtidos com o perfilômetro inercial a laser, obteve-se perfilogramas com tendência de identificar uma quantidade superior de picos de defeitos excedendo o Blanking Band que em perfis obtidos com o perfilógrafo Califórnia. Quanto maior foi o IP dos perfilogramas, o pavimento apresentou, em geral, maior quantidade de defeitos, acontecendo também uma redução do desvio percentual entre as magnitudes dos defeitos identificados pelos dois equipamentos.

Observa-se também a tendência do perfilômetro inercial a laser construir perfis longitudinais de forma mais detalhada que o perfilógrafo Califórnia. Isto se deve, provavelmente, ao primeiro equipamento empregar uma taxa de aquisição de dados elevada, com 4.000 pps (pontos/segundo).

Como os levantamentos foram realizados a uma velocidade média de tráfego de $40 \mathrm{~km} / \mathrm{h}$, a distância entre leituras foi de aproximadamente $2,8 \mathrm{~mm}$, o que tornou possível utilizar um comprimento de onda dos defeitos $(\lambda)$ a partir de $5 \mathrm{~mm}$. Outro fato a ser considerado é que o mesmo realiza uma medida sem contato, o que permite um melhor delineamento do perfil, identificando defeitos de poucos centímetros. Os mesmos podem não ser bem detalhados no perfilógrafo Califórnia, devido o pneu da ponta de prova não ter acesso a pequenas aberturas. Estes fatores isoladamente, ou em conjunto, tornam possível reproduzir melhor as características dos defeitos da superfície do pavimento pelo perfilômetro inercial a laser.

Observou-se uma tendência de convergência entre os perfilogramas resultantes dos levantamentos com os dois equipamentos estudados. Portanto, os comprimentos de onda que exercem influência na irregularidade longitudinal são identificados satisfatoriamente por ambos os equipamentos.

Nos testes de hipótese realizados, não se pôde rejeitar a hipótese nula de que as variâncias e médias dos valores de IP e IRI obtidos a partir de monitoramentos com os dois equipamentos são iguais. Portanto, constituem-se de dados obtidos de uma mesma população.

Com isto, o equipamento perfilômetro inercial a laser pode ser uma alternativa para monitoramento de perfis lon- 
gitudinais para pavimento em CCP, com objetivo de obtenção de perfilogramas e índices que servem de critério para aceitação de pavimento recém-construído. Também há viabilidade técnica para monitoramento de rodovias em operação, quando o objetivo também é a obtenção de índices de irregularidade longitudinal.

\section{REFERÊNCIAS}

ASTM (1999a) ASTM E1926-98 - Standard Practice for Computing International Roughness Index of Roads from Longitudinal Profile Measurements. American Society of Testing and Materials, Estados Unidos.

ASTM (1999b) ASTM E950-98 - Standard Test Method for Measuring the Longitudinal Profile of Traveled Surfaces with an Accelerometer Establishes Inertial Profiling Reference. American Society of Testing and Materials, Estados Unidos.

ASTM (2012) ASTM E1274-03 - Standard test method for measuring pavements roughness using a profilograph. American Society of Testing and Materials, Estados Unidos.

ASTM (2014) ASTM E2955-13 - Standard Practice for Simulating Profilograph Response to Longitudinal Profiles of Traveled Surfaces. American Society of Testing and Materials, Estados Unidos.

Barella, R. M (2008) Contribuição para a avaliação da irregularidade longitudinal de pavimentos com perfilômetros inerciais. Tese de Doutorado. Escola Politécnica da Universidade de São Paulo. São Paulo.

Benevides, S. A. S e Motta, L. M. G (2010) Comparação dos valores de irregularidade longitudinal medidos com o perfilômetro a laser com variação do arranjo das posições dos sensores. Revista Transportes, v. XVIII, n. 2, p. 72-80. DOI: $10.14295 /$ transportes.v18i2.426

Bernucci, L. B.; Ceratti, J. A. P.; Motta, L. M. G.; Soares, J. B. (2006) Pavimentação asfáltica: Formação básica para engenheiros. Rio de Janeiro: PETROBRAS: ABEDA.

Bottura, E. J. (1998) Contribuição para o estudo da calibração e controle de sistemas medidores de irregularidade rodoviária tipo resposta. Tese de Doutorado, Escola Politécnica da Universidade de São Paulo, São Paulo.

Bowman, B. L.; Ellen, B. P e Gardiner, M. S. (2002) Evaluation of pavement smoothness reduction and pay factor determination for Alabama department of transportation. Final Report on Alabama Department of Transportation - Research Project 930371. Auburn.

CALIFORNIA TEST 526 (2012) Method of Test for Operation of California Highway Profilograph and Evaluation of Profiles. Department of Transportation Division of Engineering Services, Estados Unidos.

Cantisani, G. e Loprencipe, G (2010) Road Roughness and Whole Body Vibration: Evaluation Tools and Comfort Limits. Journal of Transportation Engineering, Vol. 136, No. 9, (c)ASCE, ISSN 0733-947X/2010/9-818,

http://dx.doi.org/10.1061/(ASCE)TE.1943-5436.0000143

Carey, JR. e Irick, P. E. (1960) The pavement serviceabilityperformance concept. HRB Buletin $\mathrm{n}^{\circ} 250$.

Chou, S.F e Pellinen, T. K (2005) Assessment of Construction Smoothness Specification Pay Factor Limits Using Artificial
Neural Network Modeling. Journal of Transportation Engineering, Vol. 131, No. 7, (A)ASE, ISSN 0733947X/2005/7-563-570, http://dx.doi.org/10.1061/(ASCE)0733947X(2005)131:7(563)

Divinsky, M (2000) Repeatability Analysis of Road Roughness Measurements. Journal of Testing and Evaluation, JTEVA, Vol. 28, No. 5, p. 378-394, DOI: 10.1520/JTE12126J

DNIT (2013) DNIT 049/2013 - ES: Execução de Pavimento Rígido com Equipamento de Fôrma-Deslizante. Departamento Nacional de Infra-Estrutura de Transportes, Rio de Janeiro.

DNIT (2004) DNIT 62/2004 - PRO: Pavimento Rígido Avaliação Objetiva. Departamento Nacional de Infraestrutura de Transportes, Rio de Janeiro.

DNIT (2004) DNIT 63/2004 - PRO: Pavimento Rígido Avaliação Subjetiva. Departamento Nacional de Infraestrutura de Transportes, Rio de Janeiro.

Kim, S., Ceylan, H. e Gopalakrishnan, K. (2008) Smoothness Variations of Early-Age Jointed Plain Concrete Pavements. Canadian Journal of Civil Engineering, Vol. 35, No. 12, p. 13881398. DOI: 10.1139/L08-086

Kim, S.; Rhee, S.; Park, H. e Yun, D. (2009) Correlations among Pavement Surface Roughness, Moving Dynamic Vehicle Loads, and Concrete Pavement Performance. Performance Modeling and Evaluation of Pavement Systems and Materials: p. 25-31. DOI: $10.1061 / 4104$ perera7(354)4

Ksaibati, K. A, S. e Adkins, T. M. (1993). Factors affecting repeatability of pavement longitudinal profile measurements. Transportation Research Record 1410, Transportation Research Board, Washington, DC, p. 59-66.

Lee, D. G, (2007) Dynamic Prediction Model of As-Built Roughness in Asphaltic Concrete Pavement Construction. Journal of Transportation Engineering, Vol. 133, No. 2, February 1, 2007. (CASCE, ISSN 1943-5436, DOI: 10.1061/(ASCE)0733-947X(2007)133:2(90)

Liu, C.; Wang Z. e Lee, J. N. (2008) Influence of concrete joints on roughness index and pavement serviceability. International Journal of Pavement Research and Technology, ISSN 19971400 , v. 1 , n. 4 , p. 143-147. DOI: 10.1080/14680629.2008.9690110

Nakahara, S. M.; L. e Balbo, J. T. (2006) Desenvolvimento de modelos de previsão de irregularidade através da análise de medidas repetidas. Anais do XX Congresso de Pesquisa e Ensino em Transportes. Rio de Janeiro: Associação Nacional de Pesquisa e Ensino em Transportes, 2006. v. II. p. 1329-1340.

Paterson, W. D. O. (1987) Road Deterioration and Maintenance Effects. The Highway Design and Maintenance Standards Series, Washington D.C.

Perera, R. W. e Kohn, S. D. (2002) Issues in Pavement Smoothness. National Cooperative Highway Research Program Web Document 42. Transportation Research Board, National Research Council, Washington, DC.

Perera, R. W. e Kohn, S. D. (2005) Achieving a high level of smoothness in concrete pavements without sacrificing long-term performance. Final Report to Federal Highway Administration, No. FHWA-HRT-05-069. 
Sandberg, U. e Ejsmont, J. (2002) Tyre/Road Noise Reference

Book. INFORMEX Ejsmont Sandberg Handelsbolag. Kisa:

Sweden.

Sayers, M. W.; Karamihas, S. M. (1998) The Little Book of

Profiling. University of Michigan. Disponível em:

http://www.umtri.umich.edu/content/LittleBook98R.pdf. Acesso em dezembro de 2014

Sayers, M. W., Gillespie, T. D., Paterson, W. D. O. (1986)

Guidelines for conducting and calibrating road roughness measurements. World Bank technical paper $n^{\circ} 46$, ISSN 02537494.

Siddique, Z.; Hossain, M.; e Parcells, W (2007) Effect of Curing on Roughness Development of Concrete Pavements. Journal of Materials in Civil Engineering, 575-582. DOI:

10.1061/(ASCE)0899-1561(2007)19:7(575)

Smith, K. L.; Smith, K. D.; Evans, L. D.; Hoerner, T. E. e Darter, M. (1996) Smoothness specifications for pavements. Final Rep. to National Cooperative Highway Research Program (NCHRP) 1-31, Transportation Research Board, Washington, D.C.

Smith, K. L.; Glover L. T.; Evans L. D. (2002) Pavement Smoothness Index Relationships, FHWA-RD-02-057.

Washington, D.C.

Soncim, S. P.; Fernandes Júnior, J. L. e Campos, L. E. P. (2013)

Modelo de desempenho de irregularidade longitudinal desenvolvido com base em dados da rede de rodovias em tratamento superficial duplo do Estado da Bahia. Revista Transportes, v. 21, n. 3. DOI: 10.4237/transportes.v21i3.681

Wang, H. (2006) Road Profiler Performance Evaluation and Accuracy Criteria Analysis: Thesis Master of Science. Faculty of Virginia Polytechnic Institute and State University Blacksburg. Virginia.

Wen, H. e Chen, C. (2007) Factors Affecting Initial Roughness of Concrete Pavement. Journal of Performance of Constructed Facilities. Vol. 21, No. 6, 459-464. DOI: 10.1061/(ASCE)08873828(2007)21:6(459)

Wen, H.; Titi, H. e Berry, D. (2005) Study of rutting and roughness in asphalt overlay and local calibration of roughness prediction model in 2002 design guide. 84th Transportation Research Board Annual Meeting, National Research Council, Washington, D.C. 